\title{
Galerkin least squares finite element method for the obstacle problem
}

\author{
Erik Burman, ${ }^{\text {a }}$ Peter Hansbo, ${ }^{\mathrm{b}}$ Mats G. Larson, ${ }^{\mathrm{c}}$ \\ Rolf Stenberg ${ }^{\mathrm{d}}$ \\ ${ }^{a}$ Department of Mathematics, University College London, London, UK-WC1E \\ $6 B T, U K$ \\ ${ }^{\mathrm{b}}$ Department of Mechanical Engineering, Jönköping University, SE-55111 \\ Jönköping, Sweden \\ ${ }^{\mathrm{c}}$ Department of Mathematics and Mathematical Statistics, Umeå University, \\ SE-901 87 Umeå, Sweden \\ ${ }^{\mathrm{d}}$ Institute of Mathematics, Helsinki University of Technology, P. O. Box 1100, \\ 02015 TKK, Finland
}

\begin{abstract}
We construct a consistent multiplier free method for the finite element solution of the obstacle problem. The method is based on an augmented Lagrangian formulation in which we eliminate the multiplier by use of its definition in a discrete setting. We prove existence and uniqueness of discrete solutions and optimal order a priori error estimates for smooth exact solutions. Using a saturation assumption we also prove an a posteriori error estimate. Numerical examples show the performance of the method and of an adaptive algorithm for the control of the discretization error.
\end{abstract}

Key words: Obstacle problem, augmented Lagrangian method, a priori error estimate, a posteriori error estimate, adaptive method

\section{Introduction}

Our aim in this paper is to design a simple consistent penalty method for contact problems that avoids the solution of variational inequalities. We eliminate the need for Lagrange multipliers to enforce the contact conditions by using its definition in a discrete setting, following an idea of Chouly and Hild [4] used for elastic contact. 


\subsection{The model problem}

We consider the obstacle problem of finding the displacement $u$ of a membrane constrained to stay above an obstacle given by $\psi=\psi(x, y)$ (with $\psi \leq 0$ at $\partial \Omega)$ :

$$
\begin{aligned}
-\Delta u-f & \geq 0 \text { in } \Omega \subset \mathbb{R}^{2} \\
u & \geq \psi \text { in } \Omega \\
(u-\psi)(f+\Delta u) & =0 \text { in } \Omega \\
u & =0 \text { on } \partial \Omega
\end{aligned}
$$

where $\Omega$ is a convex polygon. It is well known that this problem admits a unique solutions $u \in H^{1}(\Omega)$. This follows from the theory of Stampacchia applied to the corresponding variational inequality (see for instance [8]).

\subsection{The finite element method}

There exists a large body of literature treating finite element methods for unilateral problems in general and obstacle problems in particular, e.g., $11,13,6,10,8,3,16,18,2,17$, . Discretization of (1) is usually performed directly starting from the variational inequality or using a penalty method. The first approach however leads to some nontrivial choices in the construction of the discretization spaces in order to satisfy the nonpenetration condition and associated inf-sup conditions and until recently it has proved difficult to obtain optimal error estimates [9,5]. The latter approach, o $\mathrm{n}$ the other hand leads to the usual consistency and conditioning problems of penalty methods.

An alternative is to use the augmented Lagrangian method. We introduce the Lagrange multiplier $\lambda$ such that

$$
\begin{aligned}
-\Delta u+\lambda & =f \text { in } \Omega \\
u & =0 \text { on } \partial \Omega
\end{aligned}
$$

under the Kuhn-Tucker side conditions

$$
\begin{aligned}
\psi-u & \leq 0 \text { in } \Omega \\
\lambda & \leq 0 \text { in } \Omega \\
(\psi-u) \lambda & =0 \text { on } \Omega .
\end{aligned}
$$


Using the standard trick of rewriting the Kuhn-Tucker conditions as

$$
\lambda=-\frac{1}{\gamma}[\psi-u-\gamma \lambda]_{+}
$$

where $[x]_{ \pm}= \pm \max (0, \pm x)$ and $\gamma \in \mathbb{R}^{+}$, cf., e.g., Chouly and Hild [4], we can formulate the augmented Lagrangian problem of finding $(u, \lambda)$ that are stationary points to the functional

$$
\begin{aligned}
\mathfrak{F}(u, \lambda):= & \frac{1}{2} \int_{\Omega}|\nabla u|^{2} d \Omega+\int_{\Omega} \frac{1}{2 \gamma}[\psi-u-\gamma \lambda]_{+}^{2} d \Omega \\
& -\frac{1}{2} \int_{\Omega} \gamma \lambda^{2} d \Omega-\int_{\Omega} f u d \Omega,
\end{aligned}
$$

cf. Alart and Curnier [1], leading to seeking $(u, \lambda) \in H_{0}^{1}(\Omega) \times L_{2}(\Omega)$ such that

$$
\int_{\Omega} \nabla u \cdot \nabla v d \Omega-\int_{\Omega} \frac{1}{\gamma}[\psi-u-\gamma \lambda]_{+} v d \Omega=\int_{\Omega} f v d \Omega \quad \forall v \in H_{0}^{1}(\Omega)
$$

and

$$
\int_{\Omega} \frac{1}{\gamma}[\psi-u-\gamma \lambda]_{+} \mu d \Omega+\int_{\Omega} \lambda \mu d \Omega=0 \quad \forall \mu \in L_{2}(\Omega) .
$$

For our discrete method, we assume that $\{\mathcal{T}\}_{h}$ is a family of conforming shape regular meshes on $\Omega$, consisting of triangles $\mathcal{T}=\{T\}$ and define $V_{h}$ as the space of $H^{1}$-conforming piecewise polynomial functions on $\mathcal{T}$, satisfying the homogeneous boundary condition of $\Gamma_{D}$.

$$
V_{h}:=\left\{v_{h} \in H_{0}^{1}(\Omega):\left.v_{h}\right|_{T} \in \mathbb{P}_{k}(T), \forall T \in \mathcal{T}\right\}, \quad \text { for } k \geq 2 .
$$

We then formally replace $\lambda$ element-wise by $\Delta u_{h}+f$ to obtain a discrete minimization problem: seek $u_{h} \in V_{h}$ such that

$$
u_{h}=\arg \min _{v \in V_{h}} \mathfrak{F}_{h}(v)
$$

where

$$
\begin{aligned}
\mathfrak{F}_{h}(v):= & \frac{1}{2} \int_{\Omega}|\nabla v|^{2} d \Omega+\sum_{T \in \mathcal{T}} \int_{T} \frac{1}{2 \gamma}[\psi-v-\gamma(\Delta v+f)]_{+}^{2} d \Omega \\
& -\frac{1}{2} \sum_{T \in \mathcal{T}} \int_{T} \gamma(\Delta v+f)^{2} d \Omega-\int_{\Omega} f v d \Omega .
\end{aligned}
$$

The Euler-Lagrange equations corresponding to (9) take the form: Find $u_{h} \in$ $V_{h}$ such that

$$
a\left(u_{h}, v_{h}\right)+b\left(u_{h}, \psi, f ; v_{h}\right)=\left(f, v_{h}\right)_{\Omega} \quad \forall v_{h} \in V_{h}
$$

where $(\cdot, \cdot)_{\Omega}$ denotes the standard $L^{2}$-inner product, $a\left(u_{h}, v_{h}\right):=\left(\nabla u_{h}, \nabla v_{h}\right)_{\Omega}$ 
and

$$
\begin{aligned}
b\left(u_{h}, \psi, f ; v_{h}\right):= & \left\langle\gamma^{-1}\left[\psi-u_{h}-\gamma\left(\Delta u_{h}+f\right)\right]_{+},-v_{h}-\gamma \Delta v_{h}\right\rangle_{h} \\
& -\left\langle\gamma\left(\Delta u_{h}+f\right), \Delta v_{h}\right\rangle_{h}
\end{aligned}
$$

where

$$
\left\langle x_{h}, y_{h}\right\rangle_{h}:=\sum_{T \in \mathcal{T}} \int_{T} x_{h} y_{h} \mathrm{~d} x
$$

and, for use below,

$$
\left\|x_{h}\right\|_{h}:=\left\langle x_{h}, x_{h}\right\rangle_{h}^{1 / 2} .
$$

To simplify the notation below we introduce $P_{\gamma}\left(u_{h}\right)=\gamma \Delta u_{h}+u_{h}$ and

$$
b\left(u_{h}, \psi, f ; v_{h}\right):=\left\langle\gamma^{-1}\left[\psi-\gamma f-P_{\gamma}\left(u_{h}\right)\right]_{+},-P_{\gamma}\left(v_{h}\right)\right\rangle_{h}-\left\langle\gamma\left(\Delta u_{h}+f\right), \Delta v_{h}\right\rangle_{h} .
$$

We will also omit $\psi$ and $f$ from the argument of $b$ below, and use the notation $\Psi:=\psi-\gamma f$ so that

$$
b\left(u_{h} ; v_{h}\right):=\left\langle\gamma^{-1}\left[\Psi-P_{\gamma}\left(u_{h}\right)\right]_{+},-P_{\gamma}\left(v_{h}\right)\right\rangle_{h}-\left\langle\gamma\left(\Delta u_{h}+f\right), \Delta v_{h}\right\rangle_{h} .
$$

Note that the form $b$ can be interpreted as a nonlinear consistent least squares penalty term for the imposition of the contact condition. A similar method was proposed in Stenberg et al. [7] in the framework of variational inequalities.

We will below alternatively use the compact notation

$$
A_{h}\left(u_{h}, v_{h}\right):=a\left(u_{h}, v_{h}\right)+b\left(u_{h} ; v_{h}\right)
$$

and the associated formulation, find $u_{h} \in V_{h}$ such that

$$
A_{h}\left(u_{h} ; v_{h}\right)=\left(f, v_{h}\right)_{\Omega}, \text { for all } v_{h} \in V_{h} \text {. }
$$

\subsection{Summary of main results and outline}

In Section 2 we recall some technical results, in Section 3 we derive an existence result for the discrete solution using Brouwer's fixed point theorem and we prove uniqueness of the solution using monotinicity of the the nonlinearity, in Section 4 we prove an a priori error estimate and using a saturation assumption we also prove an a posteriori error estimate, finally in Section 5 we present numerical results confirming our theoretical results and illustrating the performance of an adaptive algorithm based on our a posteriori error estimate. 


\section{Technical results}

Below we will use the notation $a \lesssim b$ for $a \leq C b$ where $C$ is a constant independent of $h$, but not of the local mesh geometry.

First we recall the following inverse inequality,

$$
\left\|\nabla v_{h}\right\|_{T} \leq C_{i} h_{T}^{-1}\left\|v_{h}\right\|_{T}, \quad T \in \mathcal{T}
$$

see Thomée [15].

We will use the Scott-Zhang interpolant preserving boundary conditions, denoted $i_{h}: H_{0}^{1}(\Omega) \mapsto V_{h}$. This operator is $H^{1}$-stable, $\left\|i_{h} u\right\|_{H^{1}(\Omega)} \lesssim\|u\|_{H^{1}(\Omega)}$ and the following interpolation error estimate is known to hold [14],

$$
\left\|u-i_{h} u\right\|_{\Omega}+h\left\|u-i_{h} u\right\|_{H^{1}(\Omega)}+h^{2}\left\|\Delta\left(u-i_{h} u\right)\right\|_{h} \lesssim h^{k+1}|u|_{H^{k+1}(\Omega)} .
$$

The essential properties of the nonlinearity are collected in the following lemmas.

Lemma 1 Let $a, b \in \mathbb{R}$ then there holds

$$
\begin{gathered}
\left([a]_{+}-[b]_{+}\right)^{2} \leq\left([a]_{+}-[b]_{+}\right)(a-b), \\
\left|[a]_{+}-[b]_{+}\right| \leq|a-b| .
\end{gathered}
$$

PROOF. Developing the left hand side of the expression we have

$$
[a]_{+}^{2}+[b]_{+}^{2}-2[a]_{+}[b]_{+} \leq[a]_{+} a+[b]_{+} b-a[b]_{+}-[a]_{+} b=\left([a]_{+}-[b]_{+}\right)(a-b) .
$$

For the proof of the second claim, this is trivially true in case both $a$ and $b$ are positive or negative. If $a$ is negative and $b$ positive then

$$
\left|[a]_{+}-[b]_{+}\right|=|b| \leq|b-a|
$$

and similarly if $b$ is negative and $a$ positive

$$
\left|[a]_{+}-[b]_{+}\right|=|a| \leq|b-a|
$$

Lemma 2 (Continuity of $b(\cdot ; \cdot)$ ) For all $u_{1}, u_{2}, v \in V_{h}$, the form (11) satisfies $\left|b\left(u_{1} ; v\right)-b\left(u_{2} ; v\right)\right| \lesssim \gamma^{-1}\left(\left\|\left(u_{1}-u_{2}\right)\right\|_{\Omega}+\gamma h^{-1}\left\|\nabla\left(u_{1}-u_{2}\right)\right\|_{\Omega}\right)\left(\|v\|_{\Omega}+\gamma h^{-1}\left\|\nabla v_{h}\right\|_{\Omega}\right)$. 


\section{PROOF.}

$$
\begin{aligned}
b\left(u_{1} ; v_{h}\right)-b\left(u_{2} ; v_{h}\right)= & \gamma^{-1}\left\langle\left[\Psi-P_{\gamma}\left(u_{1}\right)\right]_{+}-\left[\Psi-P_{\gamma}\left(u_{2}\right)\right]_{+},-P_{\gamma}\left(v_{h}\right)\right\rangle_{h} \\
& -\left\langle\gamma \Delta\left(u_{1}-u_{2}\right), \Delta v_{h}\right\rangle_{h} .
\end{aligned}
$$

Using the second inequality of Lemma 1 we see that the nonlinearity satisfies

$$
\begin{aligned}
\gamma^{-1} \mid\left\langle\left[\Psi-P_{\gamma}\left(u_{1}\right)\right]_{+}-\right. & {\left.\left[\Psi-P_{\gamma}\left(u_{2}\right)\right]_{+},-P_{\gamma}\left(v_{h}\right)\right\rangle_{h} \mid } \\
& \leq \gamma^{-1}\left\|P_{\gamma}\left(u_{1}\right)+\Psi-P_{\gamma}\left(u_{2}\right)-\Psi\right\|_{C, f}\left\|P_{\gamma}\left(v_{h}\right)\right\|_{h} .
\end{aligned}
$$

By the inverse inequality (13) we have

$$
\left\langle\gamma \Delta\left(u_{1}-u_{2}\right), \Delta v_{h}\right\rangle_{h} \leq C_{i}^{2} \gamma h^{-2}\left\|\nabla\left(u_{1}-u_{2}\right)\right\|_{\Omega}\left\|\nabla v_{h}\right\|_{\Omega}
$$

and

$$
\left\|P_{\gamma}\left(v_{h}\right)\right\|_{h} \leq\left\|v_{h}\right\|_{\Omega}+C_{i} \gamma h^{-1}\left\|\nabla v_{h}\right\|_{\Omega}
$$

Collecting (16), (17) and (18) we have

$$
\begin{aligned}
\left|b\left(u_{1} ; v_{h}\right)-b\left(u_{2} ; v_{h}\right)\right| \leq & \left(\left\|u_{1}-u_{2}\right\|_{\Omega}+C_{i} \gamma h^{-1}\left\|\nabla\left(u_{1}-u_{2}\right)\right\|_{\Omega}\right)\left(\left\|v_{h}\right\|_{\Omega}+C_{i} \gamma h^{-1}\left\|\nabla v_{h}\right\|_{\Omega}\right) \\
& +C_{i}^{2} \gamma h^{-2}\left\|\nabla\left(u_{1}-u_{2}\right)\right\|_{\Omega}\left\|\nabla v_{h}\right\|_{\Omega}
\end{aligned}
$$

and the claim follows.

\section{Existence of unique discrete solution}

In the previous works on Nitsche's method existence and uniqueness has been proven by using the monotonicity and hemi-continuity of the operator. Here we propose a different approach where we use the Brouwer's fixed point theorem to establish existence and the monotonicity of the nonlinearity for uniqueness. We start by showing some positivity results and a priori bounds. Since we are interested in existence and uniqueness for a fixed mesh parameter $h$, we do not require that the bounds in this section are uniform in $h$.

Lemma 3 Let $u_{1}, u_{2} \in V_{h}$ and assume that

$$
\gamma<C_{i}^{-2} h^{2} / 2
$$

then there holds

$$
\begin{aligned}
\frac{\alpha}{2}\left\|u_{1}-u_{2}\right\|_{H^{1}(\Omega)}^{2}+\gamma^{-1} \|\left[\Psi-P_{\gamma}\left(u_{1}\right)\right]_{+} & -\left[\Psi-P_{\gamma}\left(u_{2}\right)\right]_{+} \|_{h}^{2} \\
& \leq A_{h}\left(u_{1} ; u_{1}-u_{2}\right)-A_{h}\left(u_{2} ; u_{1}-u_{2}\right)
\end{aligned}
$$


and

$$
\frac{\alpha}{4}\left\|u_{1}\right\|_{H^{1}(\Omega)}^{2} \leq A_{h}\left(u_{1} ; u_{1}\right)+C \alpha^{-1} \gamma^{-2}\left\|[\Psi]_{+}\right\|_{\Omega}^{2}
$$

PROOF. First observe consider the form $b(\cdot ; \cdot)$,

$$
\begin{aligned}
& b\left(u_{1} ; v_{h}\right)-b\left(u_{2} ; v_{h}\right) \\
& \qquad \gamma^{-1}\left\langle\left[\Psi-P_{\gamma}\left(u_{1}\right)\right]_{+}-\left[\Psi-P_{\gamma}\left(u_{2}\right)\right]_{+},-v_{h}-\gamma \Delta v_{h}+\Psi-\Psi\right\rangle_{h} \\
& -\left\langle\gamma \Delta\left(u_{1}-u_{2}\right), \Delta v_{h}\right\rangle_{h} .
\end{aligned}
$$

Using the monotonicity of Lemma 1 we may write

$$
\begin{gathered}
b\left(u_{1} ; u_{1}-u_{2}\right)-b\left(u_{2} ; u_{1}-u_{2}\right) \geq \gamma^{-1}\left\|\left[\Psi-P_{\gamma}\left(u_{1}\right)\right]_{+}-\left[\Psi-P_{\gamma}\left(u_{2}\right)\right]_{+}\right\|_{h}^{2} \\
-\gamma\left\|\Delta\left(u_{1}-u_{2}\right)\right\|_{h}^{2} .
\end{gathered}
$$

Observe that using an inverse inequality (13) we have

$$
\gamma\left\|\Delta\left(u_{1}-u_{2}\right)\right\|_{h}^{2} \leq C_{i} \gamma h^{-2}\left\|\nabla\left(u_{1}-u_{2}\right)\right\|_{\Omega}^{2}
$$

. We may then write

$$
\begin{array}{r}
\left(1-C_{i}^{2} h^{-2} \gamma\right)\left\|\nabla\left(u_{1}-u_{2}\right)\right\|_{\Omega}^{2}+\gamma^{-1}\left\|\left[\Psi-P_{\gamma}\left(u_{1}\right)\right]_{+}-\left[\Psi-P_{\gamma}\left(u_{2}\right)\right]_{+}\right\|_{h}^{2} \\
\leq A_{h}\left(u_{1} ; u_{1}-u_{2}\right)-A_{h}\left(u_{2} ; u_{1}-u_{2}\right)
\end{array}
$$

It follows that choosing $\gamma<C_{i}^{-2} h^{2} 2$ and applying the Poincaré inequality

$$
\alpha^{\frac{1}{2}}\|u\|_{H^{1}(\Omega)} \leq\|\nabla u\|_{\Omega}, \quad \forall u \in H_{0}^{1}(\Omega)
$$

there holds

$$
\begin{aligned}
\frac{\alpha}{2}\left\|u_{1}-u_{2}\right\|_{H^{1}(\Omega)}^{2}+\gamma^{-1} \|\left[\Psi-P_{\gamma}\left(u_{1}\right)\right]_{+} & -\left[\Psi-P_{\gamma}\left(u_{2}\right)\right]_{+} \|_{h}^{2} \\
& \leq A_{h}\left(u_{1} ; u_{1}-u_{2}\right)-A_{h}\left(u_{2} ; u_{1}-u_{2}\right) .
\end{aligned}
$$

The second inequality follows by taking $u_{2}=0$ above and noting that then

$$
\begin{aligned}
& \frac{\alpha}{2}\left\|u_{1}\right\|_{H^{1}(\Omega)}^{2}+\gamma^{-1}\left\|\left[\Psi-P_{\gamma}\left(u_{1}\right)\right]_{+}-[\Psi]_{+}\right\|_{h}^{2} \\
& \quad \leq A_{h}\left(u_{1} ; u_{1}\right)-\gamma^{-1}\left\langle[\Psi]_{+},-P_{\gamma}\left(u_{1}\right)\right\rangle_{h} \\
& \quad \leq A_{h}\left(u_{1} ; u_{1}\right)+\left(1+C_{i} \gamma h^{-1}\right) \alpha^{-\frac{1}{2}} \gamma^{-1}\left\|[\Psi]_{+}\right\|_{\Omega} \alpha^{\frac{1}{2}}\left\|u_{1}\right\|_{H^{1}(\Omega)}
\end{aligned}
$$

where we used (18) in the last step. Considering the condition on $\gamma$ and using an arithmetic-geometric inequality we may conclude.

Proposition 4 Assume that $\gamma$ saisfy (19). Then formulation (12) using the contact operator (11), admits a unique solution. 
PROOF. The uniqueness is an immediate consequence of Lemma 3. If $u_{1}$ and $u_{2}$ both are solution to (12), then

$$
A_{h}\left(u_{1} ; u_{1}-u_{2}\right)-A_{h}\left(u_{2} ; u_{1}-u_{2}\right)=\left(f, u_{1}-u_{2}\right)_{\Omega}-\left(f, u_{1}-u_{2}\right)_{\Omega}=0
$$

and we conclude that $\left\|u_{1}-u_{2}\right\|_{H^{1}(\Omega)}=0$ and hence $u_{1} \equiv u_{2}$. Let $N_{V}$ denote the number of degrees of freedom of $V_{h}$.

Consider the mapping $G: \mathbb{R}^{N_{V}} \mapsto \mathbb{R}^{N_{V}}$ defined by

$$
(G(U), V)_{\mathbb{R}^{N_{V}}}:=A_{h}\left(u_{h}, v_{h}\right)-\left(f, v_{h}\right)_{\Omega},
$$

where $U=\left\{u_{i}\right\}_{i=1}^{N_{V}}, V=\left\{v_{i}\right\}_{i=1}^{N_{V}}$, where $\left\{u_{i}\right\}$ and $\left\{v_{i}\right\}$ denotes the vectors of unknown associated to the basis functions of $V_{h}$.

By the second claim of Lemma 3, there holds

$$
\frac{\alpha}{4}\left\|u_{h}\right\|_{H^{1}(\Omega)}^{2}-C \alpha^{-1} \gamma^{-2}\left\|[\Psi]_{+}\right\|_{\Omega}^{2}-\left(f, u_{h}\right)_{\Omega} \leq A_{h}\left(u_{h}, u_{h}\right)-\left(f, u_{h}\right)_{\Omega}=(G(U), U)_{\mathbb{R}^{N_{V}}} .
$$

Since

$$
\frac{\alpha}{4}\left\|u_{h}\right\|_{H^{1}(\Omega)}^{2}-\left(f, u_{h}\right)_{\Omega} \geq \frac{\alpha}{8}\left\|u_{h}\right\|_{H^{1}(\Omega)}^{2}-C \frac{1}{\alpha}\|f\|_{\Omega}^{2}
$$

we have that for any fixed $h$ the following positivity holds for $U$ sufficiently large

$$
0<\frac{\alpha}{8}\left\|u_{h}\right\|_{H^{1}(\Omega)}^{2}-\frac{C}{\alpha}\left(\gamma^{-2}\left\|[\Psi]_{+}\right\|_{\Omega}^{2}+\|f\|_{\Omega}^{2}\right) \leq(G(U), U)_{\mathbb{R}^{N_{V}}} .
$$

Assume that this positivity holds whenever $|U| \geq q \in \mathbb{R}_{+}$. Denote by $B_{q}$ the (closed) ball in $\mathbb{R}^{N_{V}}$ with radius $q$ and assume that there is no $U \in B_{q}$ such that $G(U)=0$. Define the function

$$
\phi(U)=-q G(U) /|G(U)| .
$$

Then $\phi: B_{q} \mapsto B_{q}, \phi$ is continuous by Lemma 2 and the assumption that $|G(U)|>0$ for all $U \in B_{q}$. Hence there exists a fixed point $X \in B_{q}$ such that

$$
X=\phi(X) .
$$

It follows that

$$
|X|^{2}=-q(G(X), X) /|G(X)|
$$

but since $|X|=q$, by assumption $(G(X), X)>0$, which leads to a contradiction, since $|X|>0$. 


\section{Error estimates}

Theorem 5 (A priori error estimate) Assume that $u \in H_{0}^{1}(\Omega)$ with $\Delta u \in$ $L^{2}(\Omega)$ is the solution of (11) and that $u_{h}$ is the solution to (10) with (11) and $0<\gamma=\gamma_{0} h^{2}$, where $\gamma_{0} \in \mathbb{R}, \gamma_{0}<C_{i}^{-2} / 2$. then there holds for all $v_{h} \in V_{h}$

$$
\begin{aligned}
\alpha\left\|u-u_{h}\right\|_{H^{1}(\Omega)}^{2}+\gamma^{-1}\left\|\left[\Psi-P_{\gamma}\left(u_{h}\right)\right]_{+}-\left[\Psi-P_{\gamma}(u)\right]_{+}\right\|_{h}^{2} \\
\quad \lesssim \frac{1}{\alpha}\left\|u-v_{h}\right\|_{H^{1}(\Omega)}^{2}+\left\|\gamma^{-\frac{1}{2}}\left(u-v_{h}\right)\right\|_{\Omega}+\left\|\gamma^{\frac{1}{2}} \Delta\left(u-v_{h}\right)\right\|_{h}^{2} .
\end{aligned}
$$

If in addition $u \in H^{k+1}(\Omega)$ then there holds

$$
\alpha\left\|u-u_{h}\right\|_{H^{1}(\Omega)}+\gamma^{-1 / 2}\left\|\left[\Psi-P_{\gamma}\left(u_{h}\right)\right]_{+}-\left[\Psi-P_{\gamma}(u)\right]_{+}\right\|_{h} \lesssim h^{k}|u|_{H^{k+1}(\Omega)} .
$$

PROOF. Using the definition of $a(\cdot, \cdot)$ we may write

$$
\begin{aligned}
\left\|\nabla\left(u-u_{h}\right)\right\|_{\Omega}^{2} & \leq a\left(u-u_{h}, u-u_{h}\right) \\
& =a\left(u-u_{h}, u-v_{h}\right)+a\left(u-u_{h}, v_{h}-u_{h}\right) \\
& \leq \frac{\alpha}{4}\left\|u-u_{h}\right\|_{H^{1}(\Omega)}^{2}+\frac{1}{\alpha}\left\|u-v_{h}\right\|_{H^{1}(\Omega)}^{2}+a\left(u-u_{h}, v_{h}-u_{h}\right) .
\end{aligned}
$$

Observe that

$$
\begin{aligned}
a\left(u, v_{h}-u_{h}\right) & =\left\langle-\Delta u-f+f, v_{h}-u_{h}\right\rangle_{V^{\prime}, V} \\
& =\left\langle\gamma^{-1}\left[\Psi-P_{\gamma}(u)\right]_{+},\left(v_{h}-u_{h}\right)\right\rangle_{V^{\prime}, V}+\left(f, v_{h}-u_{h}\right)_{\Omega}
\end{aligned}
$$

If $\left[\Psi-P_{\gamma}(u)\right]_{+} \in L^{2}(\Omega)$ we may also write

$$
\left\langle\Delta u+f, \gamma \Delta\left(v_{h}-u_{h}\right)\right\rangle_{h}+\left\langle\gamma^{-1}\left[\Psi-P_{\gamma}(u)\right]_{+}, \gamma \Delta\left(v_{h}-u_{h}\right)\right\rangle_{h}=0 .
$$

It follows that

$$
\begin{aligned}
a\left(u, v_{h}-u_{h}\right)= & \left(f, v_{h}-u_{h}\right)_{\Omega}-\left\langle\gamma^{-1}\left[\Psi-P_{\gamma}(u)\right]_{+},-P_{\gamma}\left(v_{h}-u_{h}\right)\right\rangle_{h} \\
& +\left\langle\Delta u+f, \gamma \Delta\left(v_{h}-u_{h}\right)\right\rangle_{h} \\
= & \left(f, v_{h}-u_{h}\right)_{\Omega}-b\left(u ; v_{h}-u_{h}\right) .
\end{aligned}
$$


As a consequence we have the following property reminiscent of Galerkin orthogonality,

$$
\begin{aligned}
a(u- & \left.u_{h}, v_{h}-u_{h}\right) \\
= & b\left(u_{h} ; v_{h}-u_{h}\right)-b\left(u ; v_{h}-u_{h}\right) \\
= & \left\langle\gamma^{-1}\left[\Psi-P_{\gamma}\left(u_{h}\right)\right]_{+}-\gamma^{-1}\left[\Psi-P_{\gamma}(u)\right]_{+},-P_{\gamma}\left(v_{h}-u_{h}\right)\right\rangle_{h} \\
\quad & \quad-\gamma\left\langle\Delta\left(u_{h}-u\right), \Delta\left(v_{h}-u_{h}\right)\right\rangle_{h}
\end{aligned}
$$

First observe that

$$
\begin{aligned}
\gamma\langle\Delta & \left.\left(u_{h}-u\right), \Delta\left(v_{h}-u_{h}\right)\right\rangle_{h} \\
& \leq\left\|\gamma^{\frac{1}{2}}\left(\Delta u_{h}-\Delta v_{h}\right)\right\|_{h}^{2}+\left\|\gamma^{\frac{1}{2}}\left(\Delta v_{h}-\Delta u\right)\right\|_{h}\left\|\gamma^{\frac{1}{2}}\left(\Delta v_{h}-\Delta u_{h}\right)\right\|_{h} \\
& \leq \frac{1}{2} C_{i}^{2} h^{-2} \gamma\left\|\nabla\left(u_{h}-v_{h}\right)\right\|_{\Omega}^{2}+\left\|\gamma^{\frac{1}{2}}\left(\Delta v_{h}-\Delta u\right)\right\|_{h}^{2} \\
& \leq C_{i}^{2} h^{-2} \gamma\left\|\nabla\left(u_{h}-u\right)\right\|_{\Omega}^{2}+C_{i}^{2} h^{-2} \gamma\left\|\nabla\left(v_{h}-u\right)\right\|_{\Omega}^{2}+\left\|\gamma^{\frac{1}{2}}\left(\Delta v_{h}-\Delta u\right)\right\|_{h}^{2} .
\end{aligned}
$$

Considering the first term in the right hand side of equation (25) we may write

$$
\begin{aligned}
\left\langle\gamma^{-1}\left[\Psi-P_{\gamma}\left(u_{h}\right)\right]_{+}-\gamma^{-1}\left[\Psi-P_{\gamma}(u)\right]_{+},-P_{\gamma}\left(v_{h}-u_{h}\right)\right\rangle_{h} \\
=\underbrace{\left\langle\gamma^{-1}\left[\Psi-P_{\gamma}\left(u_{h}\right)\right]_{+}-\gamma^{-1}\left[\Psi-P_{\gamma}(u)\right]_{+},-P_{\gamma}\left(v_{h}-u\right)\right\rangle_{h}}_{I} \\
\quad+\underbrace{\left\langle\gamma^{-1}\left[\Psi-P_{\gamma}\left(u_{h}\right)\right]_{+}-\gamma^{-1}\left[\Psi-P_{\gamma}(u)\right]_{+},-P_{\gamma}\left(u-u_{h}\right)\right\rangle_{h}}_{I I} \\
=I+I I .
\end{aligned}
$$

The term I may be bounded using the Cauchy-Schwarz inequality followed by the arithmetic geometric inequality

$$
I \leq \epsilon \gamma^{-1}\left\|\left[\Psi-P_{\gamma}\left(u_{h}\right)\right]_{+}-\left[\Psi-P_{\gamma}(u)\right]_{+}\right\|_{h}^{2}+\frac{1}{4 \epsilon}\left\|\gamma^{-\frac{1}{2}} P_{\gamma}\left(v_{h}-u\right)\right\|_{h}^{2} .
$$

For the term $I I$ we use the monotonicity property $\left([a]_{+}-[b]_{+}\right)(b-a) \leq$ $-\left([a]_{+}-[b]_{+}\right)^{2}$, with $a=\Psi-P_{\gamma}\left(u_{h}\right)$ and $b=\Psi-P_{\gamma}(u)$ so that

$\left([a]_{+}-[b]_{+}\right)(b-a)=\left(\left[\Psi-P_{\gamma}\left(u_{h}\right)\right]_{+}-\gamma^{-1}\left[\Psi-P_{\gamma}(u)\right]_{+}\right)\left(\Psi-P_{\gamma}(u)-\Psi+P_{\gamma}\left(u_{h}\right)\right)$

to deduce that

$$
I I \leq-\gamma^{-1}\left\|\left[\Psi-P_{\gamma}\left(u_{h}\right)\right]_{+}-\left[\Psi-P_{\gamma}(u)\right]_{+}\right\|_{h}^{2} .
$$


Collecting the above bounds and using the Poincaré inequality (20) we find,

$$
\begin{aligned}
& \alpha\left(\frac{3}{4}-C_{i}^{2} h^{-2} \gamma\right)\left\|u-u_{h}\right\|_{H^{1}(\Omega)}^{2} \\
& +(1-\epsilon) \gamma^{-1}\left\|\left[\Psi-P_{\gamma}\left(u_{h}\right)\right]_{+}-\left[\Psi-P_{\gamma}(u)\right]_{+}\right\|_{h}^{2}+(1-\epsilon)\left\|\gamma^{\frac{1}{2}} \Delta\left(u-u_{h}\right)\right\|_{h}^{2} \\
& \quad \leq \frac{1}{\alpha}\left\|u-v_{h}\right\|_{H^{1}(\Omega)}^{2}+\frac{1}{4 \epsilon}\left\|\gamma^{-\frac{1}{2}} P_{\gamma}\left(u-v_{h}\right)\right\|_{h}^{2}+\left\|\gamma^{\frac{1}{2}} \Delta\left(u-v_{h}\right)\right\|_{h}^{2}
\end{aligned}
$$

Fixing $\epsilon=\frac{1}{2}$, and fixing $\gamma$ sufficiently small so that $C_{i}^{2} h^{-2} \gamma \leq \alpha / 4$ then there holds

$$
\begin{aligned}
\alpha\left\|u-u_{h}\right\|_{H^{1}(\Omega)}^{2} \gamma^{-1}\left\|\left[\Psi-P_{\gamma}\left(u_{h}\right)\right]_{+}-\left[\Psi-P_{\gamma}(u)\right]_{+}\right\|_{h}^{2} & \\
& \lesssim \frac{1}{\alpha}\left\|u-v_{h}\right\|_{H^{1}(\Omega)}^{2}+\left\|\gamma^{-\frac{1}{2}}\left(u-v_{h}\right)\right\|_{h}^{2}+\left\|\gamma^{\frac{1}{2}} \Delta\left(u-v_{h}\right)\right\|_{h}^{2} .
\end{aligned}
$$

This concludes the proof of (21). The error estimate (22) then follows by choosing $v_{h}$ to be the interpolant, $i_{h} u$, applying the approximation error estimate (14) on the form

$$
\begin{gathered}
\left\|u-i_{h} u\right\|_{H^{1}(\Omega)}+\left\|\gamma^{-\frac{1}{2}}\left(u-i_{h} u\right)\right\|_{h}+\left\|\gamma^{\frac{1}{2}} \Delta\left(u-i_{h} u\right)\right\|_{h} \\
\lesssim\left(h^{k}+\gamma^{-1 / 2} h^{k+1}+\gamma^{1 / 2} h^{k-1}\right)|u|_{H^{k+1}(\Omega)}
\end{gathered}
$$

and using the bound on $\gamma$.

Assumption: (Saturation) We assume that there exists a constant $C_{s}$ such that

$$
\left\|\Delta\left(u-u_{h}\right)\right\|_{h} \leq C_{s} h^{-1}\left\|\nabla\left(u-u_{h}\right)\right\|_{\Omega}
$$

Theorem 6 (A posteriori error estimate) Assume that $u \in H_{0}^{1}(\Omega)$ with $\Delta u \in$ $L^{2}(\Omega)$ is the solution of (11) and $u_{h}$ the solution of (10) satisfying (28) and with the parameter $\gamma$ satisfying $\gamma \leq \frac{1}{2} C_{s}^{-2} h^{2}$, then

$$
\alpha\left\|u-u_{h}\right\|_{H^{1}(\Omega)}+\gamma^{-1}\left\|\left[\Psi-P_{\gamma}\left(u_{h}\right)\right]_{+}-\left[\Psi-P_{\gamma}(u)\right]_{+}\right\|_{h} \lesssim E\left(h, \gamma, u_{h}, f\right),
$$

where

$$
E\left(h, \gamma, u_{h}, f\right):=h\left\|f+\Delta u_{h}+\gamma^{-1}\left[\Psi-P_{\gamma}\left(u_{h}\right)\right]_{+}\right\|_{h}+\left\|h^{\frac{1}{2}} \llbracket \partial_{n} u_{h} \rrbracket\right\|_{\mathcal{F}} .
$$


PROOF. Let $e=u-u_{h}$ then, under the assumption (28) and using (3) we may write

$$
\begin{aligned}
\left(1-C_{s}^{2} h^{-2} \gamma\right)\|\nabla e\|_{\Omega}^{2}+\gamma^{-1}\left\|\left[\Psi-P_{\gamma}(u)\right]_{+}-\left[\Psi-P_{\gamma}\left(u_{h}\right)\right]_{+}\right\|_{h}^{2} & \\
\leq & \left(\nabla\left(u-u_{h}\right), \nabla e\right)_{\Omega} \\
& +\gamma^{-1}\left\langle\left[\Psi-P_{\gamma}(u)\right]_{+}-\left[\Psi-P_{\gamma}\left(u_{h}\right)\right]_{+},-P_{\gamma}(e)\right\rangle_{h} \\
& -\gamma\left\langle\Delta\left(u-u_{h}\right), \Delta e\right\rangle_{h}
\end{aligned}
$$

Now, using similar arguments as in Theorem 5 we deduce

$$
(\nabla u, \nabla e)_{\Omega}-\gamma^{-1}\left\langle\left[\Psi-P_{\gamma}(u)\right]_{+}, P_{\gamma}(e)\right\rangle_{h}-\gamma\langle\Delta u, \Delta e\rangle_{h}=\left\langle f, P_{\gamma}(e)\right\rangle_{h} .
$$

Therefore the bound (30) may be written, under the assumption $C_{s}^{2} h^{-2} \gamma \leq$ $1 / 2$,

$$
\begin{aligned}
\frac{1}{2}\|\nabla e\|_{\Omega}^{2} & +\gamma^{-1}\left\|\left[\Psi-P_{\gamma}(u)\right]_{+}-\left[\Psi-P_{\gamma}\left(u_{h}\right)\right]_{+}\right\|_{h}^{2} \\
\leq & \left\langle f, P_{\gamma}(e)\right\rangle_{h}-\left(\nabla u_{h}, \nabla e\right)_{\Omega} \\
& \quad+\gamma^{-1}\left\langle\left[\Psi-P_{\gamma}\left(u_{h}\right)\right]_{+}, P_{\gamma}(e)\right\rangle_{h}+\gamma\left\langle\Delta u_{h}, \Delta e\right\rangle_{h} \\
= & \left\langle f+\Delta u_{h}+\gamma^{-1}\left[\Psi-P_{\gamma}\left(u_{h}\right)\right]_{+},(I+\gamma \Delta)\left(e-i_{h} e\right)\right\rangle_{h} \\
& +\left\langle\llbracket \partial_{h} u_{h} \rrbracket, e-i_{h} e\right\rangle_{\mathcal{F}}
\end{aligned}
$$

Using that $\gamma\left\|\Delta i_{h} e\right\|_{h} \lesssim \gamma h^{-1}\left\|\nabla i_{h} e\right\|_{\Omega}$, the $H^{1}$ stability of $i_{h}$ and the definition of $\gamma$ we obtain

$$
\begin{aligned}
& \frac{1}{2}\|\nabla e\|_{\Omega}^{2}+\gamma^{-1}\left\|\left[\Psi-P_{\gamma}(u)\right]_{+}-\left[\Psi-P_{\gamma}\left(u_{h}\right)\right]_{+}\right\|_{h}^{2} \\
& \leq C(h+\left.\gamma h^{-1}+\gamma^{\frac{1}{2}}\right)\left(\left\|f+\Delta u_{h}-\gamma^{-1}\left[\Psi-P_{\gamma}\left(u_{h}\right)\right]_{+}\right\|_{h}\right. \\
& \times\left(\|\nabla e\|_{\Omega}+\gamma^{\frac{1}{2}}\|\Delta e\|_{h}\right) \\
&+C\left\|h^{\frac{1}{2}} \llbracket \partial_{n} u_{h} \rrbracket\right\|_{\mathcal{F}}\|\nabla e\|_{\Omega} \\
& \leq C(h+\left.\gamma h^{-1}+\gamma^{\frac{1}{2}}\right)\left(\| f+\Delta u_{h}-\gamma^{-1}\left[\Psi-P_{\gamma}\left(u_{h}\right)\left\|_{h}+\right\| h^{\frac{1}{2}} \llbracket \partial_{n} u_{h} \rrbracket \|_{\mathcal{F}}\right)\right. \\
& \times\left(1+C_{s} \gamma^{\frac{1}{2}} h^{-1}\right)\|\nabla e\|_{\Omega}
\end{aligned}
$$

Once again applying the assumption on $\gamma$ we obtain the bound

$$
\begin{aligned}
\|\nabla e\|_{\Omega}+\gamma^{-1} \|[\Psi & \left.-P_{\gamma}(u)\right]_{+}-\left[\Psi-P_{\gamma}\left(u_{h}\right)\right]_{+} \|_{h} \\
& \leq C h\left\|f+\Delta u_{h}-\gamma^{-1}\left[\Psi-P_{\gamma}\left(u_{h}\right)\right]_{+}\right\|_{h}+C\left\|h^{\frac{1}{2}} \llbracket \partial_{h} u_{h} \rrbracket\right\|_{\mathcal{F}} .
\end{aligned}
$$

We conclude the proof using the Poincaré inequality (20).

Remark 7 This a posteriori error estimate has the disadvantage of the saturation assumption and also that the parameter $\gamma$ depends on the constant in the saturation assumption. However as we shall see below it appears to give 
a very good representation of the $H^{1}$-error and can be used to drive adaptive refinement.

\section{$5 \quad$ Numerical examples}

\subsection{A smooth rotational symmetric exact solution}

This example, from [12], is posed on the square $\Omega=(-1,1) \times(-1,1)$ with $\psi=0$ and

$$
f=\left\{\begin{array}{cc}
-8 r_{0}^{2}\left(1-\left(r^{2}-r_{0}^{2}\right)\right) & \text { if } r \leq r_{0}, \\
-8\left(r^{2}+\left(r^{2}-r_{0}^{2}\right)\right) & \text { if } r>r_{0},
\end{array}\right.
$$

where $r=\sqrt{x^{2}+y^{2}}$ and $r_{0}=1 / 4$, and with Dirichlet boundary conditions taken from the corresponding exact solution

$$
u=\left[r^{2}-r_{0}^{2}\right]_{+}^{2} .
$$

We choose $\gamma=\gamma_{0} h^{2}$ with $\gamma_{0}=1 / 100$ and show the convergence in the $L_{2}-$ and $H^{1}$-norms in Figure 1 together with the error indicator. We remark that the smoothness of the solution precludes mesh zoning for this example, but that the indicator has the same asymptotic behaviour as the $H^{1}$ error. An elevation of the computed solution on one of the meshes in a sequence is given in Fig. 2. We note the optimal convergence of $O\left(h^{3}\right)$ in $L_{2}$ and $O\left(h^{2}\right)$ in $H^{1}$. Here we use $h=1 / \sqrt{\mathrm{NNO}}$, wehre NNO denotes the number of nodes in a uniformly refined mesh.

\subsection{A non-smooth exact solution}

This example, from [2], is posed on the L-shaped domain $\Omega=(-2,2) \times(-2,2) \backslash$ $[0,2) \times(-2,0]$ with $\psi=0$ and

$$
f(r, \varphi)=-r^{2 / 3} \sin (2 \varphi / 3)\left(\gamma^{\prime}(r) / r+\gamma^{\prime \prime}(r)\right)-\frac{4}{3} r^{-1 / 3} \gamma^{\prime}(r) \sin (2 \varphi / 3)-\gamma_{2}(r)
$$

(note the sign error in [2]), where, with $\hat{r}=2(r-1 / 4)$,

$$
\gamma_{1}(r)= \begin{cases}1, & \hat{r}<0 \\ -6 \hat{r}^{5}+15 \hat{r}^{4}-10 \hat{r}^{3}+1, & 0 \leq \hat{r}<1 \\ 0, & \hat{r} \geq 1,\end{cases}
$$




$$
\gamma_{2}(r)=\left\{\begin{array}{l}
0, r \leq 5 / 4 \\
1 \text { elsewhere }
\end{array}\right.
$$

with Dirichlet boundary conditions taken from the corresponding exact solution

$$
u(r, \varphi)=r^{2 / 3} \gamma_{1}(r) \sin (2 \varphi / 3)
$$

which belongs to $H^{5 / 3-\varepsilon}(\Omega)$ for arbitrary $\varepsilon>0$.

For this example we plot, in Fig. 3 the error on consecutive adaptively refined meshes, using the minimum meshsize as a measure of $h$. We note the suboptimal convergence and that the indicator still approximately follows the $H^{1}$ error asymptotically. In Fig. 4 we show the corresponding sequence of refined meshes, and in Fig. 5 we show an elevation of the approximate solution on the final mesh in the sequance.

\section{References}

[1] P. Alart and A. Curnier. A mixed formulation for frictional contact problems prone to Newton like solution methods. Comput. Methods Appl. Mech. Engrg., 92(3):353-375, 1991.

[2] D. Braess, C. Carstensen, and R. H. W. Hoppe. Convergence analysis of a conforming adaptive finite element method for an obstacle problem. Numer. Math., 107(3):455-471, 2007.

[3] Z. Chen and R. H. Nochetto. Residual type a posteriori error estimates for elliptic obstacle problems. Numer. Math., 84(4):527-548, 2000.

[4] F. Chouly and P. Hild. A Nitsche-based method for unilateral contact problems: numerical analysis. SIAM J. Numer. Anal., 51(2):1295-1307, 2013.

[5] G. Drouet and P. Hild. Optimal convergence for discrete variational inequalities modelling Signorini contact in 2D and 3D without additional assumptions on the unknown contact set. SIAM J. Numer. Anal., 53(3):1488-1507, 2015.

[6] R. Glowinski and P. Le Tallec. Augmented Lagrangian and operator-splitting methods in nonlinear mechanics, volume 9 of SIAM Studies in Applied Mathematics. Society for Industrial and Applied Mathematics (SIAM), Philadelphia, PA, 1989.

[7] T. Gustafsson, R. Stenberg, and J. Videman. Mixed and stabilized finite element methods for the obstacle problem. ArXiv e-prints, March 2016.

[8] J. Haslinger, I. Hlaváček, and J. Nečas. Numerical methods for unilateral problems in solid mechanics. In Handbook of numerical analysis, Vol. IV, Handb. Numer. Anal., IV, pages 313-485. North-Holland, Amsterdam, 1996. 
[9] P. Hild and Y. Renard. An improved a priori error analysis for finite element approximations of Signorini's problem. SIAM J. Numer. Anal., 50(5):2400$2419,2012$.

[10] C. Johnson. Adaptive finite element methods for the obstacle problem. Math. Models Methods Appl. Sci., 2(4):483-487, 1992.

[11] N. Kikuchi and J. T. Oden. Contact problems in elasticity: a study of variational inequalities and finite element methods, volume 8 of SIAM Studies in Applied Mathematics. Society for Industrial and Applied Mathematics (SIAM), Philadelphia, PA, 1988.

[12] R. H. Nochetto, K. G. Siebert, and A. Veeser. Pointwise a posteriori error control for elliptic obstacle problems. Numer. Math., 95(1):163-195, 2003.

[13] R. Scholz. Numerical solution of the obstacle problem by the penalty method. Computing, 32(4):297-306, 1984.

[14] L. R. Scott and S. Zhang. Finite element interpolation of nonsmooth functions satisfying boundary conditions. Math. Comp., 54(190):483-493, 1990.

[15] V. Thomée. Galerkin finite element methods for parabolic problems, volume 25 of Springer Series in Computational Mathematics. Springer-Verlag, Berlin, second edition, 2006.

[16] A. Veeser. Efficient and reliable a posteriori error estimators for elliptic obstacle problems. SIAM J. Numer. Anal., 39(1):146-167, 2001.

[17] A. Weiss and B. I. Wohlmuth. A posteriori error estimator for obstacle problems. SIAM J. Sci. Comput., 32(5):2627-2658, 2010.

[18] Y. Zhang. Multilevel projection algorithm for solving obstacle problems. Comput. Math. Appl., 41(12):1505-1513, 2001. 


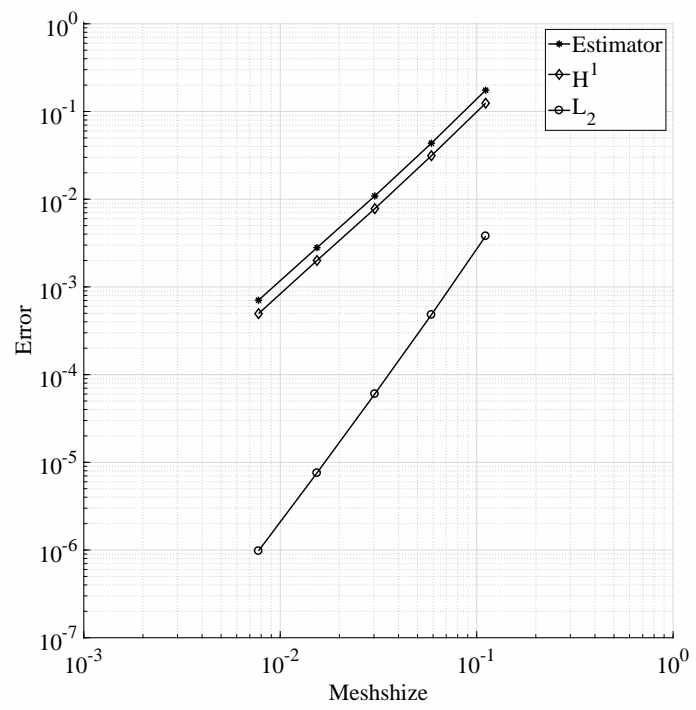

Figure 1. Convergence for the smooth case.

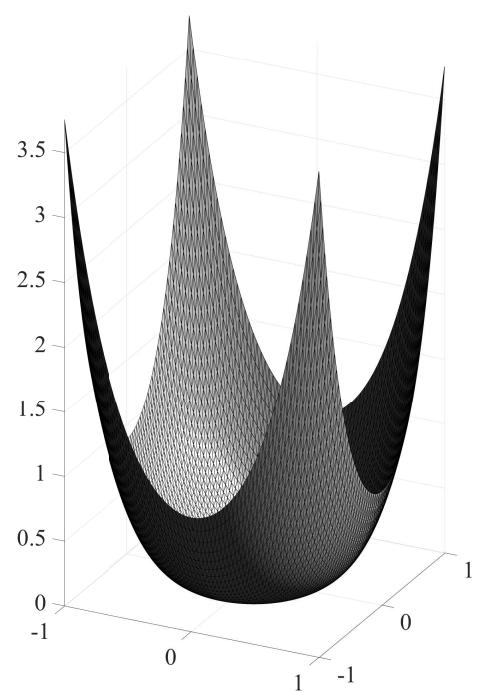

Figure 2. Elevation of the discrete solution, smooth case. 


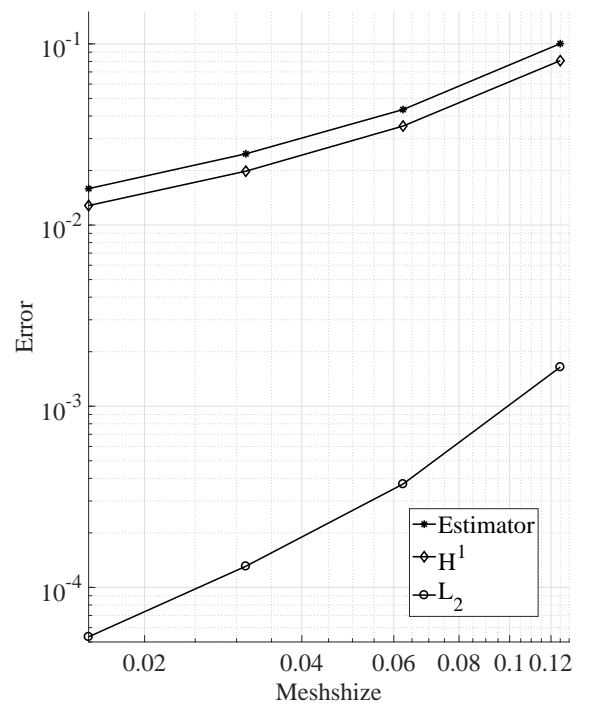

Figure 3. Convergence for the nonsmooth case.
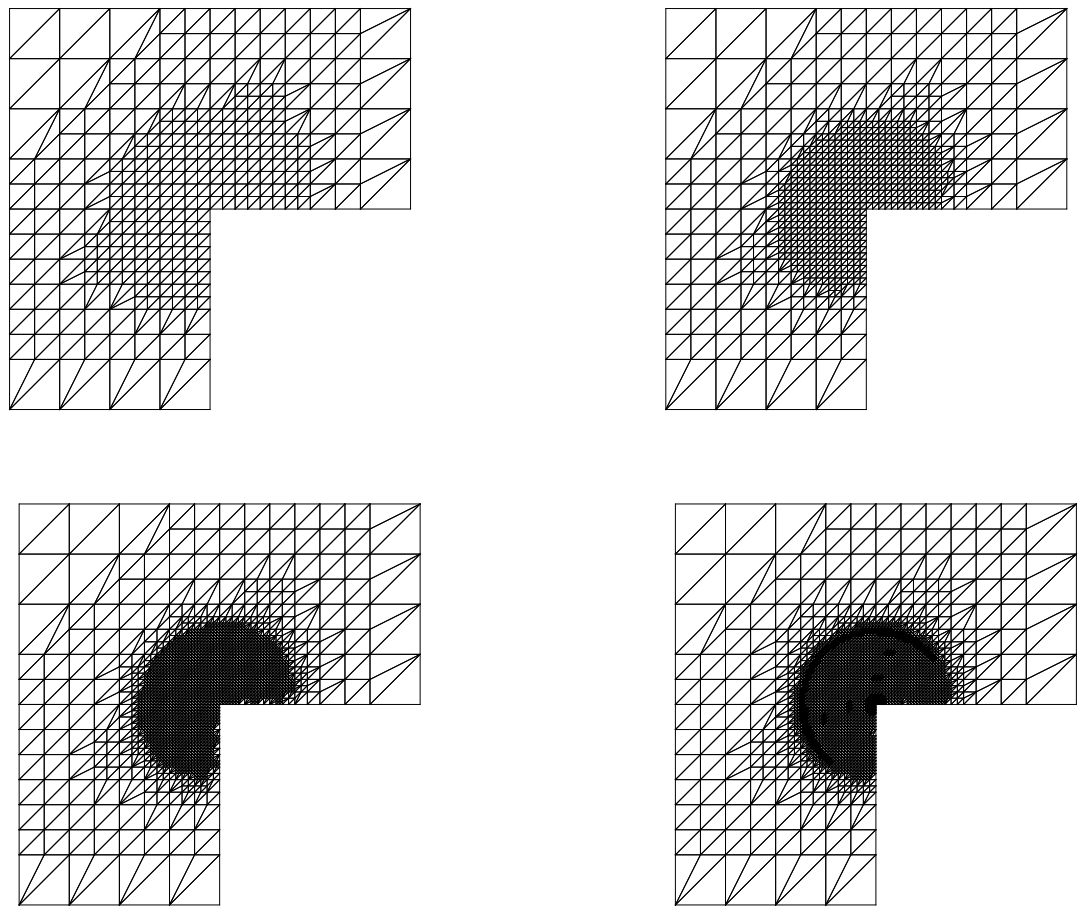

Figure 4. Sequence of refined meshes. 


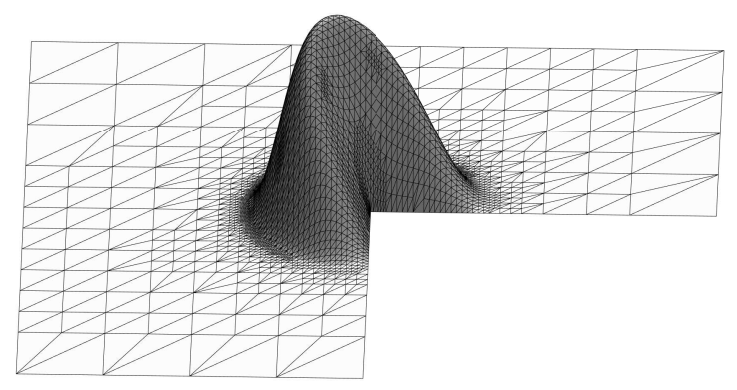

Figure 5. Elevation of the discrete solution, nonsmooth case. 